\title{
A TEM study of GaN grown by ELO on (0001) 6H-SiC
}

\author{
P. Ruterana ${ }^{1}$, B. Beaumont*, P. Gibart* and Y. Melnik**
}

Laboratoire d'Etudes et de Recherches sur les Matériaux, UPRESA 6004

CNRS, Institut de la Matière et du Rayonnement, 6 Boulevard du Maréchal Juin, 14050 Caen Cedex, France.

*Centre de Recherche sur l'Hétéroépitaxie et ses Applications, rue Bernard Grégory, Sophia Antipolis, 06560, Valbonne, France

**A.F. Ioffe Institute, St. Petersburg 194021, Russia,

present address: TDI Inc, Gaithersburg, MD 20877, USA.

1. Author for Correspondence Tel: 222314526 53, Fax: 33231452660

email: ruterana@lermat8.ismra.fr

\begin{abstract}
The misfit between $\mathrm{GaN}$ and $6 \mathrm{H}-\mathrm{SiC}$ is $3.5 \%$ instead of $16 \%$ with sapphire, the epitaxial layers have similar densities of defects on both substrates. Moreover, the lattice mismatch between $\mathrm{AlN}$ and $6 \mathrm{H}-\mathrm{SiC}$ is only $1 \%$. Therefore, epitaxial layer overgrowth (ELO) of $\mathrm{GaN}$ on AlN/6H-SiC could be a route to further improve the quality of epitaxial layers. AlN has been grown by Halide Vapour Phase Epitaxy (HVPE) on (0001) 6H-SiC, thereafter a dielectric $\mathrm{SiO}_{2}$ mask was deposited and circular openings were made by standard photolithography and reactive ion etching. We have examined GaN layers at an early stage of coalescence in order to identify which dislocations bend and try to understand why. The analysed islands have always the same hexagonal shape, limited by $\{10 \overline{10}\}$ facets. The a type dislocations are found to fold many times from basal to the prismatic plane, whereas when a+c dislocations bend to the basal plane, they were not seen to come back to a prismatic one.
\end{abstract}

\section{INTRODUCTION}

The epitaxial lateral overgrowth (ELO) technique has proven to be a relevant process to decrease the dislocation densities from $10^{10}$ to less than $10^{7} \mathrm{~cm}^{-2}$ in GaN layers grown on sapphire. It has been implemented in both Metalorganic Vapor Phase Epitaxy (MOVPE) [1-3] and Hybrid Vapor Phase Epitaxy (HVPE) $[4,5]$, and considerable attention has been focussed on it, since a blue laser diode grown on an ELO GaN substrate, resulted in a lifetime of more than 10000 hours [6]. The conventional ELO method can be 
described as follows: first, a GaN layer of a few micrometers thick is grown, then a dielectric $\left(\mathrm{SiO}_{2}\right.$ or $\left.\mathrm{Si}_{\mathrm{x}} \mathrm{N}_{\mathrm{y}}\right)$ mask is deposited. Using standard photolithographic techniques, stripes are opened in the mask. During regrowth, either by MOVPE or HVPE, growth only occurs in the openings i.e., selective area epitaxy is achieved. A lateral growth over the mask leads to full coalescence. A smooth surface suitable for device fabrication is obtained if the ratio of the lateral to vertical growth rates is high enough. The standard way to realize ELO is to induce the lateral growth from the very beginning of the regrowth process[7]. Whereas the misfit between $\mathrm{GaN}$ and $6 \mathrm{H}-\mathrm{SiC}$ is $3.5 \%$ instead of $16 \%$ on sapphire. The epitaxial layers have similar defect densities on both substrates. This effect has until now been attributed to the mosaïc growth of $\mathrm{GaN}$ on these substrates, with slightly misoriented islands bounded by threading dislocations. The lattice mismatch between AlN and $6 \mathrm{H}-\mathrm{SiC}$ is about $1 \%$ which makes it most adequate as a buffer layer for the growth of $\mathrm{GaN}$. In the following, we investigate the possibility of using HVPE AlN and 2S-ELO GaN in order to improve the quality of the active layers.

\section{EXPERIMENTAL}

Prior to epitaxy, the $\mathrm{SiC}$ was chemically cleaned and then $\mathrm{H} 2$ plasma treated, then a $100 \mathrm{~nm} \mathrm{Al}$ buffer layer was deposited by $\mathrm{HVPE}$ at $1100^{\circ} \mathrm{C}$. The growth of GaN was next performed in a homemade MOVPE vertical reactor operating at atmospheric pressure. The carrier gas used in this study is a mixture of $\mathrm{H}_{2}: \mathrm{N}_{2}$. Trimethylgallium (TMGa) and $\mathrm{NH}_{3}$ are used as precursors. A HeNe laser reflectometry set-up is used to monitor in-situ the growth process. First a $2 \mu \mathrm{m}$-thick GaN layer is deposited directly on sapphire. In order to have large areas with electron transparency, it was necessary to prepare TEM samples by the tripod method. In this technique, two specimens are glued face to face using epoxy for cross section preparation. Then the first face is polished using a series of diamond coated plastic discs down to $0.5 \mathrm{~nm}$ granulometry followed by silica colloïdal $0.05 \mathrm{~nm}$ solution. Then 0.5 to $1 \mathrm{~nm}$ slab is cut and glued on the tripod in order to grind the second face down to electron transparency. During this step, the thickness is monitored by use of silicon as one of the slabs. Therefore, when the sample thickness comes down to $10 \mu \mathrm{m}$, silicon becomes transparent and the polishing is stopped when the thickness fringes cross the interface and extend into the area of interest. In this process, the silicon is used to protect the specimen under study and is completely eliminated in the final stage of polishing. Usually a short cleaning in the ion mill is necessary before 
examination in the microscope. The specimens were analysed using a Jeol 2010 electron microscope operating at $200 \mathrm{kV}$.

\section{RESULTS}

At the initial stage of ELO, the use of circular windows has allowed us to determine the directions of fastest growth. Coalescence is only starting and the flat top islands are faceted (Figure 1a). In the Laue pattern, the mirror $\{11 \overline{2} 0\}$ planes of $\mathrm{SiC}$ show that the facets projections planes in the figure are $\{10 \overline{1} 0\}$ (figure $1 \mathrm{~b}$ ). In this figure, there is an equal probability of finding one of the $6\{10 \overline{1} 0\}$ limiting facets for each island. This type of orientation was systematically used for TEM samples in order to make observations along a $<1 \overline{2} 10>$ directions.
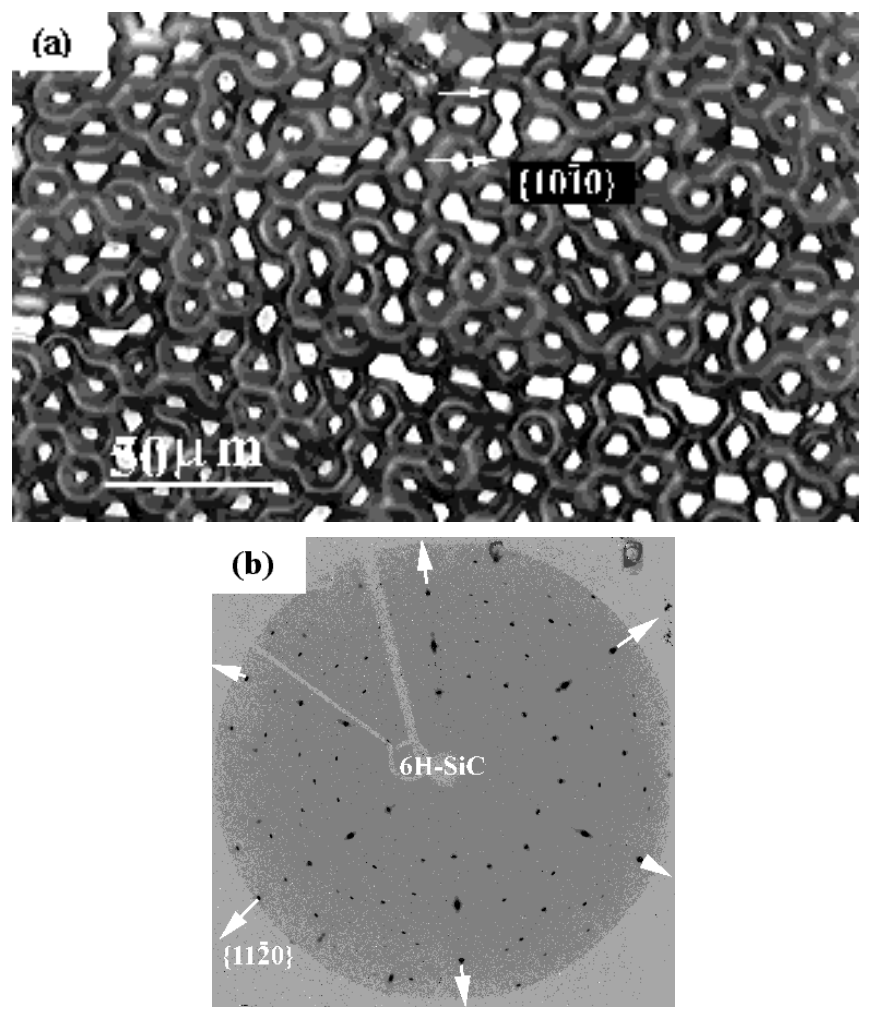

Figure 1: Surface morphology of the islands obtained by ELO during the first stage of growth (a), as well as the orientation of the surface (b). 
As seen on figure 2, the use of a tripod allows to have a transparent area which extends to more than $100 \mathrm{~nm}$; this was used to investigate the various areas of the pyramidal islands. In particular, a simple diffraction experiment confirms the Laue orientation of figure 1 and shows that the interfaces between adjacent pyramids are along vertical $\{10 \overline{1} 0\}$ facets.

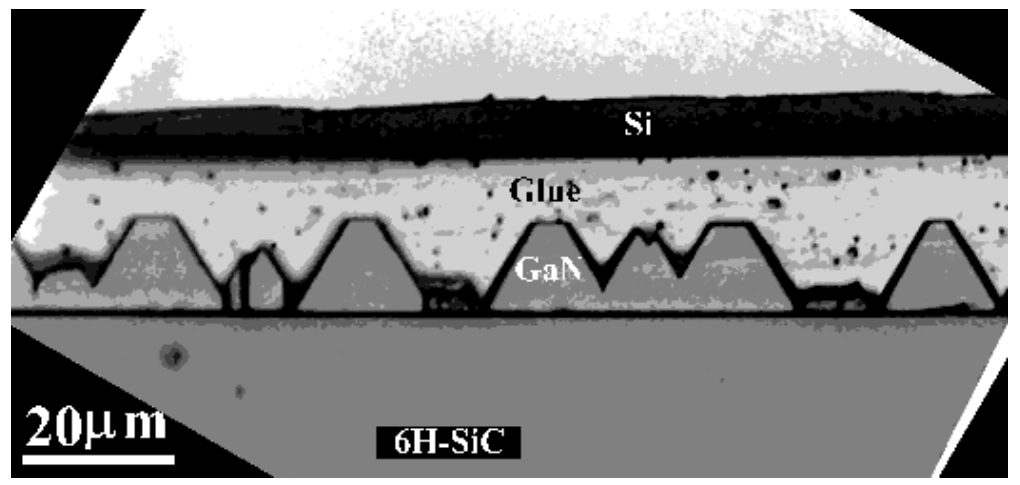

Figure 2. A TEM specimen of GaN obtained by the tripod polishing method

In particular a detailed analysis of the bending of dislocations has been carried out. From the center of the island, it can be seen in the 0002 dark field images that all the dislocations with $\mathbf{c}$ and $\mathbf{a}+\mathbf{c}$ Burgers vectors bend to the basal plane (Fig. 3). Once this bending is accomplished, the dislocation will definitely stay in the basal plane.

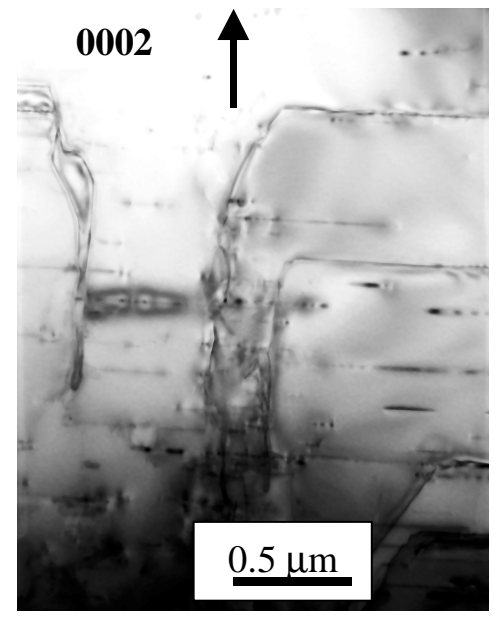

Figure 3. A 0002 weak beam image showing the bending of threading dislocations which have a c component in their Burgers vectors. 
In the case of edge type $\langle 11 \overline{2} 0\rangle$ threading dislocations, the behaviour appear to be slightly different. As shown of figure 4 , which is a $11 \overline{2} 0$ micrograph of the same area as in figure 3. This type of dislocations appear to fold many times from the basal to the prismatic plane in the center of the island. However, as one goes away of the central area, the dislocations have bent to the basal plane. This is in agreement with all the reported observations which show that ELO is able to lead to a drastic reduction of the threading dislocation densities.

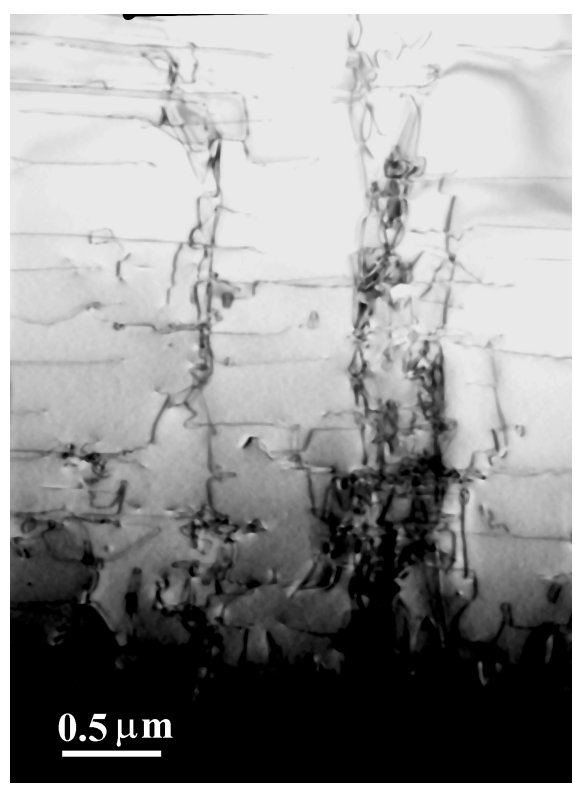

Figure 4. A $11 \overline{2} 0$ weak beam micrograph: threading dislocations with a component Burgers vectors fold more than once in basal and prismatic planes

\section{Conclusion}

In this work, we have shown that the threading dislocations bend to the basal plane on top of HVPE AIN on SiC. Based on these results and those available in the literature, it may be concluded that the lateral epitaxy is not a substrate driven mechanism. It is a consequence of the anisotropy of the properties of the crystals, such as dependence of the growth velocity on the crystallographic directions. Moreover, it is shown that the dislocation behavior is probably dependent on their Burgers vector. However more work is still needed in order to be able to make a more definite conclusion. 


\section{References}

${ }^{1}$ D. Kapolnek, S. Keller, R. Vetury, R. D. Underwood, P. Kozodoy, S. P. Denbaars, and U. K. Mishra, Appl. Phys. Lett. 71, 1204 (1997).

2 O.-H. Nam, M. D. Bremser, T. S. Zheleva, and R. F. Davis, Appl. Phys. Lett. 71, 2638 (1997).

${ }^{3}$ H. Marchand, X.H. Wu, J.P. Ibbetson, P.T. Fini, P. Kozodoy, S. Keller, J.S. Speck, S.P. DenBaars, U.K. Mishra, Appl. Phys. Lett. 73, 747 (1998).

${ }^{4}$ A. Usui, H. Sunakawa, A. Sakai, and A. A. Yamaguchi, Jpn. J. Appl. Phys. 36, L899 (1997).

${ }^{5}$ A. Sakai, H. Sunakawa, and A. Usui, Appl. Phys. Lett. 71, 2259 (1997).

6 S. Nakamura, M. Senoh, S. Nagahama, N. Iwasa, T. Yamada, T. Matsushita, H. Kiyoku, Y. Sugimoto, T. Kozaki, H. Umemoto, M. Sano, and K. Chocho, Proceedings of ICNS'97, Tokushima, Japan, edited by K. Hiramatsu (1997), p. 444 ; J. Cryst. Growth 189/190, 820 (1998).

${ }^{7}$ B. Beaumont, M. Vaille, G. Nataf, A. Bouillé, J.-C. Guillaume, P. Vennéguès, S. Haffouz, P. Gibart, MRS Internet J. Nitride Semicond. Res, Vol 3, Art 20 (1998). 